\title{
Multi-scale Reliability-Based Design Optimisation Framework for Fibre-Reinforced Composite Laminates
}

\author{
Sadik L. Omairey ${ }^{a b} *$, Peter D. Dunning ${ }^{a}$, and Srinivas Sriramula ${ }^{a}$ \\ ${ }^{\text {a }}$ School of Engineering, University of Aberdeen, United Kingdom \\ ${ }^{\mathrm{b}}$ Brunel Composites Centre, College of Engineering, Design and Physical Sciences, Brunel University London, \\ London, UK \\ * Corresponding author sadik.omairey@brunel.ac.uk
}

\begin{abstract}
A multi-scale reliability-based design optimisation (RBDO) framework is developed for unidirectional fibre reinforced polymer (FRP) composite laminates. Uncertainties occur in the multi-scale buildup of composites, presenting a challenge to reliability analysis. Thus, FRPs full benefits are often not realised, as conservative safety factors are used due to a lack of understanding of how uncertainties affect performance. A sequential optimisation strategy is developed, employing multi-scale surrogate models that replace expensive finite element analysis (FEA) to estimate homogenised properties and assess performance. This RBDO framework optimises a composite component, whilst accounting for several multi-scale uncertainties using a large representative volume element (LRVE).
\end{abstract}

Keywords: Composites; Reliability-based design optimisation; Uncertainty; Stiffness; Surrogate models.

\section{Introduction}

Composite materials are widely used in many industries, due to their high specific stiffness, strength, and the ability to tailor their structural properties. For example, these benefits are being exploited in the aerospace industry by replacing conventional metallic alloys with composites to reduce weight in many areas, including the heavily loaded primary structure of the wing, fuselage, and smaller, more lightly loaded components, such as ailerons and fairings [1]. The Airbus A350 XWB uses carbon fibre reinforced polymer (CFRP) composite material for $52 \%$ of its structure by weight [2]. However, despite their use in high-profile applications, the full potential of composite materials is often limited by conservative assumptions, such as a quasi-isotropic or "black metal" design process [3, 4].

Designing and optimising fibre reinforced composite materials is a more complicated process compared with alloys, due to their multi-material build-up nature, which introduces more design variables. For instance, designing an optimum vessel using an alloy will typically have shell thickness as the main design variable. Whereas, if a fibre reinforced laminate composite material is used, then the main design variables will at least include the number, thickness and orientations of plies, as can be seen in Figure 1. Consequently, a wider range of uncertainties is expected to feed into the structural system, undermining deterministic optimisation solutions that assume design variables are always at their mean value. These uncertain parameters are a result of complex engineering processes involved in the fabrication of composites [5], and their inherent and irreducible nature classifies them as aleatory uncertainties that can be defined in the form of a probabilistic distribution [6]. If uncertainties are not accounted for, then the performance of the optimised composite component will be nonoptimal, potentially leading to poor reliability and failure [7]. The traditional approach to account for uncertainties is to use safety factors. However, these are often derived from experience, or intuition and maybe too conservative, leading to inefficient design, or too optimistic, leading to poor reliability. 


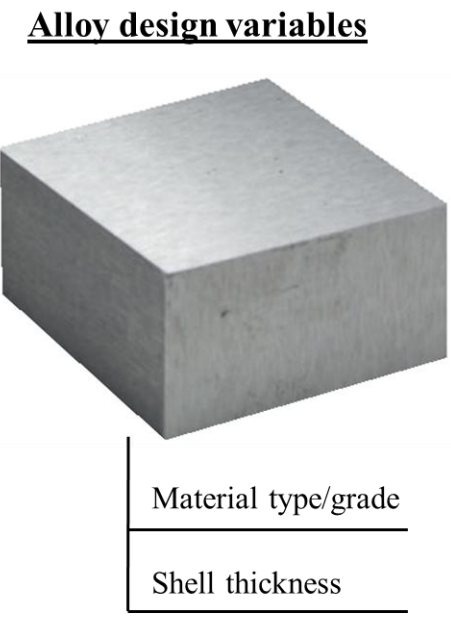

\section{Composite design variables}

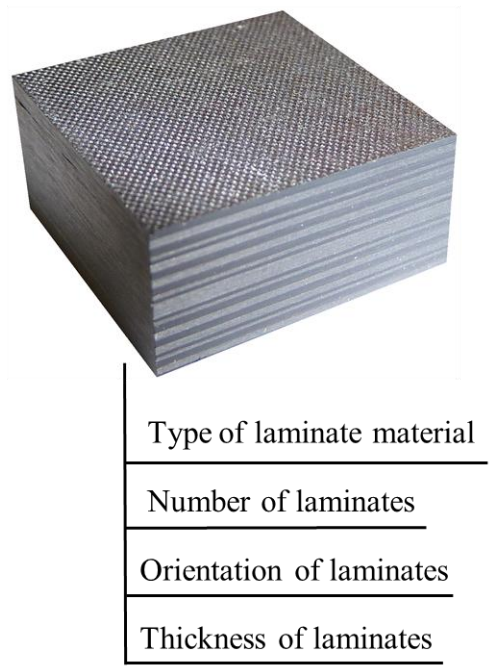

Figure 1. The main design variables of alloys and composites.

Therefore, research over the last 30 years into reliability-based design optimisation (RBDO) of structures made from composite materials has been motivated by addressing both the conservative design approach and potentially poor reliability of optimised designs. Early approaches often aimed to minimise component weight, subject to reliability constraints on failure [8,9] and/or buckling [10]. Early approaches typically used analytical models to assess the limit state function, approximate analytical reliability analysis methods, e.g. the first-order reliability method (FORM), with composite material uncertainties introduced at the ply level, e.g. ply stiffness, strength, thickness and angle. Analytical models are efficient, but limited, and cannot easily model more complex structural components. Thus, more recent approaches often use numerical methods, such as the finite element analysis (FEA), that can analyse general, complex structures, e.g. [11, 12]. The use of FORM (or similar approximate analytical methods) is also very efficient, but can be inaccurate, especially if the limit state function is highly nonlinear, or multi-modal. This has led some researchers to use Monte Carlo Simulation (MCS) for reliability computation, e.g. [8, 13, 14]. Time varying and non-probabilistic uncertainty models have also been applied to fibre-reinforced laminate structures, e.g. [15].

Also, material property uncertainties at the ply level are related to uncertainties at smaller scales. For example, ply stiffness properties in a fibre-reinforced composite are influenced by uncertainties in the fibre and matrix material properties, fibre volume ratio and fibre stacking [7, 16-20]. However, combining multi-scale modelling with RBDO for fibre-reinforced composite components has received little attention to date. Rouhi and Rais-Rohani [14] developed a multi-scale RBDO method to design a cylinder made from a nanofiberenhanced fibre-reinforced composite material. The aim was to minimise weight, subject to a buckling reliability constraint, evaluated using MCS and surrogate models. The multi-scale framework used analytical models at each scale (including the component scale), so it may not be easily applied to more general structures and limit state functions, or include some types of uncertainty, such as fibre stacking. Further examples of multi-scale RBDO for fibre-reinforced composite materials could not be found, although such approaches have been developed for other types of composite material, such as a carbon nanotube/polymer composite structure [21], hybrid composite shells [22] and multi-scale topology optimisation [23]. Thus, there is currently a lack of a general method for multi-scale RBDO of fibre-reinforced composite laminate components, which is the main contribution of this paper.

Numerical models, MCS and multi-scale uncertainty propagation all add significant computational cost. Thus, techniques have been used to improve the efficiency of RBDO method for composite materials when using these approaches. A popular approach is to create efficient surrogate models that replace expensive finite element analyses. This could be done globally, where a single surrogate model is created that uses all the design and random variables as inputs and can output the reliability, and/or objective function [14, 24]. This may be feasible for a small number of total variables, but creating surrogate models for a large number of input parameters can be computationally expensive, as a large number of training points are often needed for accuracy 
[25]. In multi-scale RBDO Ghasemi et al. [21] created surrogate models for just the multi-scale uncertainty propagation model. Another approach is to use multiple surrogate models. For example, Rais-Rohani et al. [11] compared global surrogate modelling with an approach using a series of local models, each focusing on a region around the current design point. It was concluded that the local approach is more computationally efficient than the global approach.

RBDO problems for composite materials can be solved using a double-loop approach, where the reliability analysis is performed as an inner-loop each time the design variables are updated to assess the reliability constraints [12]. However, this can result in a large number of function evaluations, leading to high computational cost $[24,26,27]$. Thus, the double loop approach is usually only computationally feasible when solving the problem using a gradient-based optimisation method, with analytical models (or small numerical models) and an approximate analytical reliability method (e.g. FORM). However, gradient-based methods can only find local optima and cannot directly include discrete design variables (such as a discrete set of ply thicknesses, or angles). Thus, some approaches have used non-gradient-based optimisation methods, such as Particle Swarm Optimisation (PSO) [28, 29] and Genetic Algorithms (GAs) [13, 24, 30] to address these issues, which also adds significant computational cost. Surrogate models can again help to reduce computational cost (especially if MCS, multi-scale analysis and large numerical models are also used).

Another strategy for RBDO is to first perform a deterministic optimisation, then analyse its probabilistic response and reliability to define a new deterministic problem with modified constraints. This reduces the number of function evaluations required to find the optimum point. This method is sometimes known as the Sequential Optimisation Reliability Analysis (SORA) strategy [31, 32], or decoupled approach [25]. In addition, surrogate modelling can be used to further reduce the computational cost of this strategy [24, 25]. The implementation of both SORA and surrogate modelling to conduct RBDO has been investigated in the literature. For example, Sohouli et al. [33] used surrogate modelling to approximate the limit state function within a decoupled RBDO framework to optimise composite structures with uncertainty in the material properties and applied loads. Another study used sequential optimisation in the form of decoupled deterministic optimisation and reliability analysis to performing RBDO of composite panels in post-buckling regime, considering the uncertainty of the elastic properties of the composite [30]. Both studies used uncertain laminate level properties without considering the multi-scale propagation of uncertainties.

The aim of this research is to create an efficient multi-scale RBDO method for fibre-reinforced composite materials, that accounts for a wide range of uncertainties (including geometric uncertainties at the fibre-matrix scale), uses MCS, to avoid issues with nonlinear and multi-modal limit state functions, a non-deterministic optimisation method, to avoid local minima and potentially include discrete design variables, and a finite element model at the component scale. This is achieved by extending and combining our previous surrogatebased framework for an off-line multi-scale uncertainty propagation [17] with an on-line sequential optimisation and reliability analysis approach and adaptive local surrogate modelling strategy. A novel feature of our multiscale framework is an inclusive FEA-based material representation unit, known as a large representative volume element (LRVE) that interpolates the effect of spatially correlated micro and meso-scale material and geometrical uncertainties, yet is computationally efficient for integration within a developed sequential RBDO framework.

Although some aspects of our proposed approach have been investigated for fibre-reinforced composite optimisation before, they have not previously been combined. In particular, there is currently no general method for multi-scale RBDO of fibre-reinforced composite laminate components. The main contribution of this research is to show that the combination of the above methods results in an approach that is general whilst also being computationally efficient.

\section{Methodology}

Performing RBDO for components that can be analysed analytically is simple compared with complex components that require numerical analysis with significant computational time. To demonstrate the feasibility of conducting a RBDO for numerically analysed components, an efficient FEA-based multi-scale reliability analysis developed by the authors $[17,18]$ is used within a sequential deterministic and probabilistic optimisation strategy to reduce the mass of a composite component, whilst meeting a certain level of stiffness reliability, considering a wide range of uncertainties. An overview of this RBDO framework can be seen in Figure 2, and the required steps are explained in the following sections. 


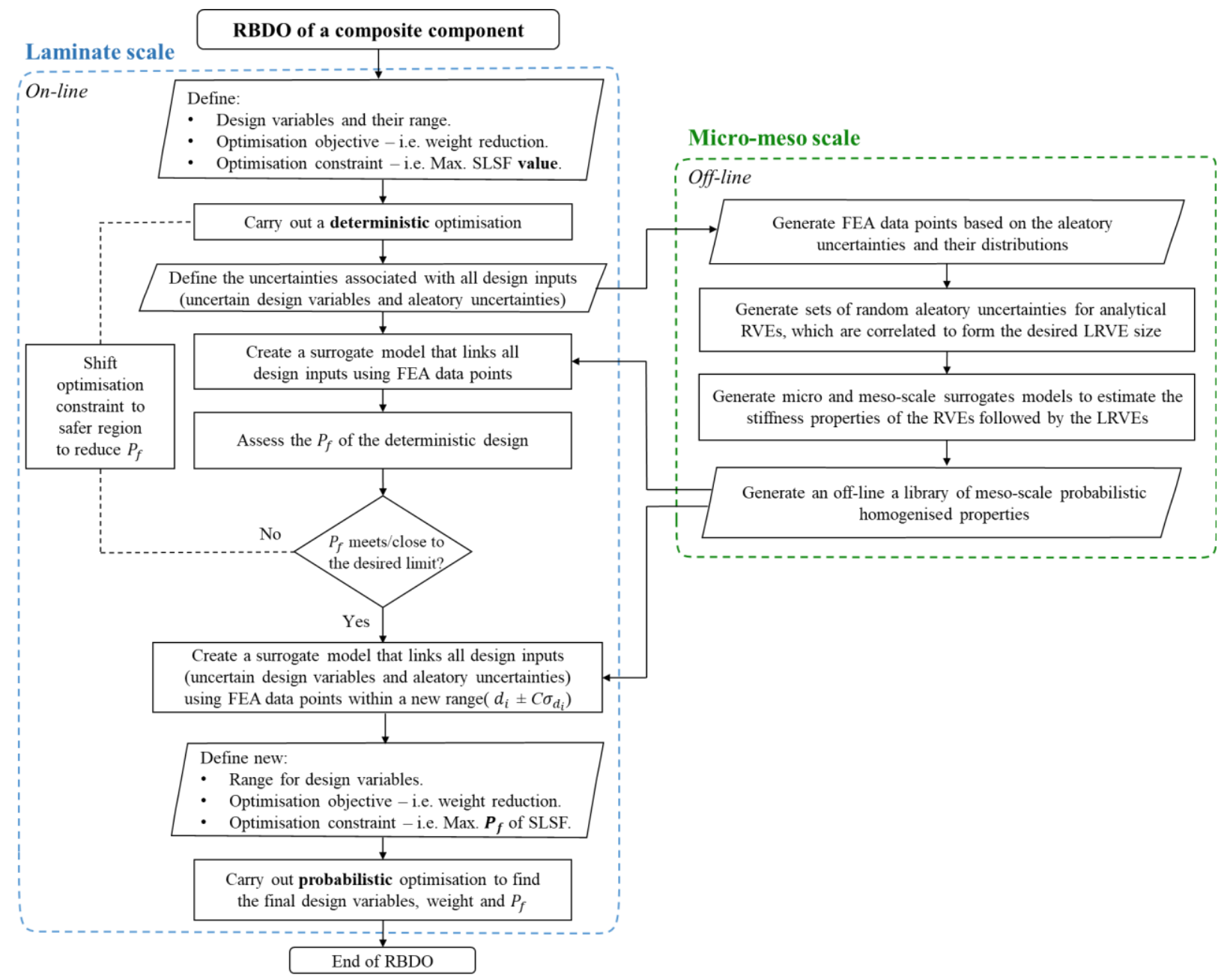

Figure 2. Proposed framework flowchart of RBDO for composite components.

\subsection{Deterministic optimisation}

In this phase, an optimisation technique is employed to find the minimum mass of a component, while meeting a certain deterministic design constraint, as shown in Eq. (1) below:

$$
\begin{array}{ll}
\text { min: } & M \\
\text { find: } & d_{i}=\text { Design variable }(i) \\
\text { subject to: } & g\left(d_{i}, x_{j}\right) \leq l \\
& \left\{d_{i} \mid d_{i}^{\text {min }} \leq d_{i} \leq d_{i}^{\text {max }}\right\} \\
& x_{j}=\mu_{x_{j}}
\end{array}
$$

Where $M$ is the component mass, $l$ is the design constraint upper limit, $d_{i}$ is a design variable and $x_{j}$ represents other (non-design variables) inputs, such as material properties and loads, assumed constant at their mean value $\left(\mu_{x_{i}}\right)$ in the deterministic optimisation problem.

As uncertainties are ignored, the optimiser can often solve the deterministic problem within an acceptable number of function evaluations and time, depending on the computational cost of each evaluation. When the optimum solution is reached, the optimum design variables will be situated close to the failure region. This is expected because all uncertain values are assumed to be at their mean during optimisation. Thus, there is a high probability of failure when uncertainties are taken into account. 


\subsection{Uncertainty propagation framework}

In order to assess the reliability of the composite component at a design point, there is a need to propagate the effect of uncertainties across the component micro and meso-scales. This is achieved by generating an offline library of probabilistic material properties (see Figure 4) using an efficient FEA-based multi-scale probabilistic framework developed by the authors [17]. This probabilistic framework is composed of FEAbased, computationally efficient surrogate models, capable of capturing the effect of various multi-scale uncertainties on the homogenised elastic properties of continuous fibre-reinforced composites. It takes the statistical information of uncertainties as an input and uses a large representative volume element (LRVE) that can model spatial variation of uncertainties, compared with a single RVE containing only one or two fibres, as shown in Figure 3. The LRVE is formed by correlating smaller RVEs in terms of material properties and geometric uncertainties, and employing a series of efficient surrogate models trained using a limited number of FE data points analysed using the periodic RVE homogenisation method [17], making it feasible to generate the large amount of probabilistic homogenised properties required to assess reliability accurately using Monte Carlo Simulation (MCS).

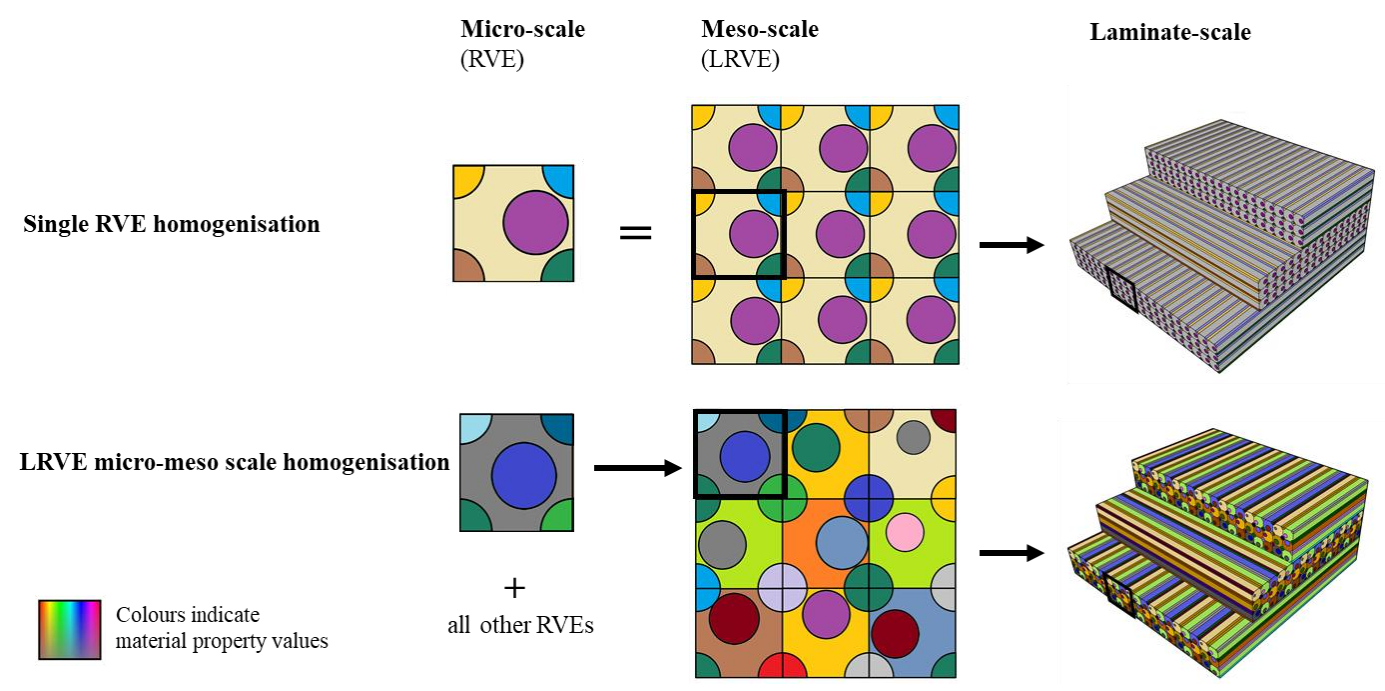

Figure 3. The homogenisation approaches employed in this study.

\subsection{Reliability assessment}

The reliability of the deterministic optimisation solution is assessed at this stage by replacing the deterministic values of the optimum design variables $\left(d_{i}\right)$ with the information on distribution type and statistical properties. Furthermore, other inputs such as material properties and load are also assigned with their statistical properties, as shown in Eq. (2) below:

$$
\begin{aligned}
& D_{i}=f\left(d_{i} \mid \mu_{d_{i}}, \sigma_{d_{i}}^{2}\right) \\
& x_{j}=f\left(x_{j} \mid \mu_{x_{j}}, \sigma_{x_{j}}^{2}\right) \\
& P_{f}=P\left[g\left(D_{i}, x_{j}\right) \leq 0\right]
\end{aligned}
$$

Where $P_{f}$ is the probability of failure of the limit state function $g\left(D_{i}, x_{j}\right)$, which is related to the structural response of the component. $D_{i}$ is a probabilistic random variable of the optimum design variable $\left(d_{i}\right)$ established in the $1^{\text {st }}$ deterministic optimisation, and $x_{j}$ represents the other uncertain inputs such as material properties and loads. Reliability is assessed using MCS where each evaluation of the limit-state function is performed by computationally cheap surrogate models, as explained next.

\subsection{Surrogate modelling}

Due to the number of probabilistic inputs $\left(D_{i}, x_{j}\right)$, there is a need to create surrogate models around the current design point to reduce the processing time required to evaluate the serviceability limit state function (SLSF). Within the proposed framework, two categories of these surrogate models are created: 1) surrogate(s) 
used to assess the reliability, and 2) a final surrogate used to assess the reliability while performing probabilistic optimisation. On top of both, are the surrogates that assess the effect of uncertainties on the homogenised elastic properties, as explained in section 2.2. An overview of these surrogates is shown in Figure 4.

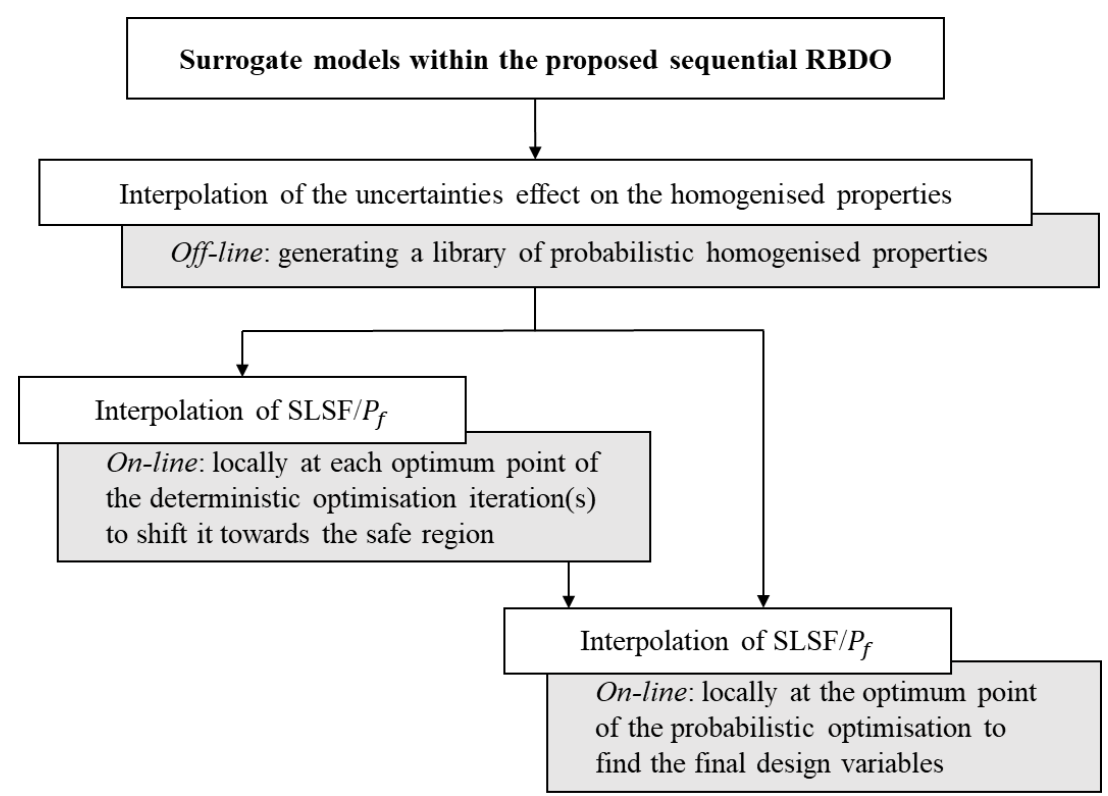

Figure 4. The categories of the surrogate models used in the proposed sequential RBDO.

The above two categories of surrogate models are trained using data points generated from analysing several randomly generated models. These FEA models are generated by assigning probability properties to all inputs. In addition to the training data points, another set of experimental data points are generated to assess the accuracy of the surrogate model. This is achieved by comparing the numerically calculated SLSF value of the experimental data point, against the value estimated from the surrogate model of the same design inputs. Then, several experimental points are used to calculate the mean value of the error, as shown in Figure 5.

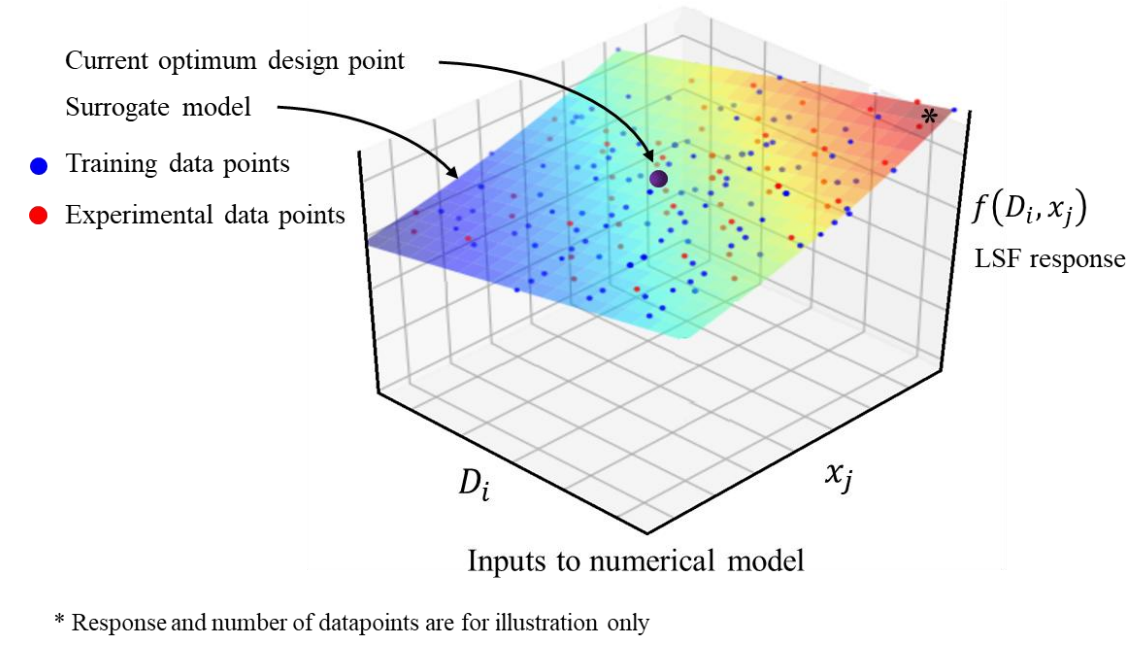

Figure 5. A representation of the training and experimental points used to create the SLSF response surrogate model.

\subsection{Probabilistic optimisation}

Once the surrogate is created and verified, the reliability at the $1^{\text {st }}$ deterministic optimum design can be assessed. If it falls below the desired limit, the design shifts towards the safe region by altering the deterministic constraint value in $(1)$. The updated constraint value $\left(l_{2}\right)$ is estimated by evaluating the inverse cumulative 
distribution function at the desired probability of failure $\left(P_{\text {limit }}\right)$. The data are fitted with a normal distribution to find the mean $\left(\mu_{\text {fitted }}\right)$ and standard deviation $\left(\sigma_{\text {fitted }}\right)$, which are used to return the inverse value as shown in Eq. (3).

$$
l_{2}=\frac{1}{\sqrt{2 \pi} \sigma_{\text {fitted }}} e^{-\frac{\left(P_{\text {limit }}-\mu_{\text {fitted }}\right)^{2}}{2 \sigma_{\text {fitted }}^{2}}}
$$

The loop of finding deterministic optimum points and assessing their reliability is repeated until the desired reliability is met, as shown in Figure 2. However, meeting the desired reliability limit is not necessarily the optimum design, as it was obtained from a deterministic optimisation. Thus, once the deterministic optimisation process finds a feasible solution, the current design variables are used as the starting point for a probabilistic optimisation (or double-loop approach). This is achieved by employing a surrogate model around the last design that met the desired reliability limit as illustrated in Eq. (4).

$$
\begin{array}{ll}
\min : & M \\
\text { find: } & D_{i}=\text { Mean design variable }(i) \\
\text { subject to: } & \left(P_{f}=P\left[g\left(D_{i}, x_{j}\right) \leq 0\right]\right) \leq P_{\text {limit }} \\
& \left\{D_{i} \mid d_{i}-C \sigma_{d_{i}} \leq D_{i} \leq d_{i}+C \sigma_{d_{i}}\right\} \\
& x_{j}=f\left(x_{j} \mid \mu_{x_{j}}, \sigma_{x_{j}}^{2}\right)
\end{array}
$$

This surrogate uses data points generated around the deterministic optimum point (that met the desired reliability) by analysing random models generated within the boundaries of $C$ times the standard deviation $\left(d_{i} \pm\right.$ $C \sigma_{d_{i}}$ ) of the design variables, as it is expected that the solution will be close to the final deterministic optimum. On the other hand, the design constraint is replaced with the maximum desired probability of failure, which is the probability of exceeding the original constraint, as defined in the original deterministic problem (1).

\section{Numerical example}

A skewed propeller blade made from a symmetric 4-ply fibre-reinforced composite material is used to demonstrate the capability of the proposed multi-scale RBDO framework. The effect of using different LRVE sizes on the optimum design is also investigated. The geometry of the propeller is attached as supplementary data.

\subsection{Design variables}

Fibre-reinforced composites are often designed and used with limited ply orientations, mostly $0{ }^{\circ}, 90^{\circ}$, $\pm 45^{\circ}$. However, allowing ply orientations to vary continuously may lead to more efficient designs, due to greater elastic tailoring capabilities [4]. Hence, in this example, both ply orientation and ply thickness are used as bounded continuous design variables, as seen in Table 1. However, because the framework can use nongradient-based methods, optimising with discrete ply angles and/or thicknesses is also possible.

\subsection{Uncertainties}

An $E$-glass fibre-epoxy composite is selected to investigate the developed framework. Uncertainties for this composite material are presented within two categories: the first category is the micro and meso scales, this includes fibre and matrix material properties, fibre stacking and fibre volume ratio $\left(V_{f}\right)$ as explained below:

Material uncertainties: Represented by varying the properties of each phase based on their statistical properties shown in Table 1. For a single RVE, this includes one matrix and five fibre sections (central fibre and four quarters) as shown in Figure 6(a).

Fibre stacking and volume ratio uncertainties: The reliability framework divides fibres into two categories: fixed fibres represented by the RVEs' four fibre corner quarters; geometrically speaking, these quarters remain in place and have the same diameter to preserve continuity with its neighbours. The second category is the central non-fixed fibres, which can move within the RVE to model stacking $\left(r\right.$ and $\theta$ ) and $V_{f}$ ratio uncertainties, without violating continuity, as can be seen in Figure 6(a). The statistical properties of $r$ and $\theta$ are shown in Table 1. 
Assigning material property uncertainties in the single RVE is relatively straight-forward as each sample in the MCS employs a separate RVE. However, in the LRVE, achieving an accurate and continuous representative element is required. Thus, RVEs that construct the LRVE are correlated by two means:

Fibre phase properties: The LRVE framework correlates neighbouring RVEs by assigning the same fibre material property values to all adjacent corner fibre sections, creating a type of relationship between individual RVEs and their surroundings. This can be seen in the colour mapping in Figure 6(b).

Matrix phase property uncertainties: It is unrealistic to have sharp changes in the property values of matrix material surrounding fibre filaments, as it is a continuous media. Hence, the LRVE framework smooths out matrix material stiffness property randomness using an image processing concept.

The algorithm used to implement fibre stacking uncertainty, fibre volume ratio $\left(V_{f}\right)$ and matrix properties blurring are attached in Appendices A and B. Additionally, comprehensive details on the implementation of these uncertainties can be found in our previous work [17].

The second category is within the laminate scale; it includes ply thickness, ply orientation and loading uncertainties. The properties and uncertainties used in this example are shown in Table 1, where the distributions are similar to those used in previous studies [34-37].

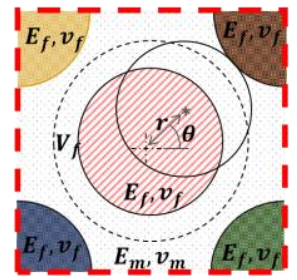

a) Single RVE

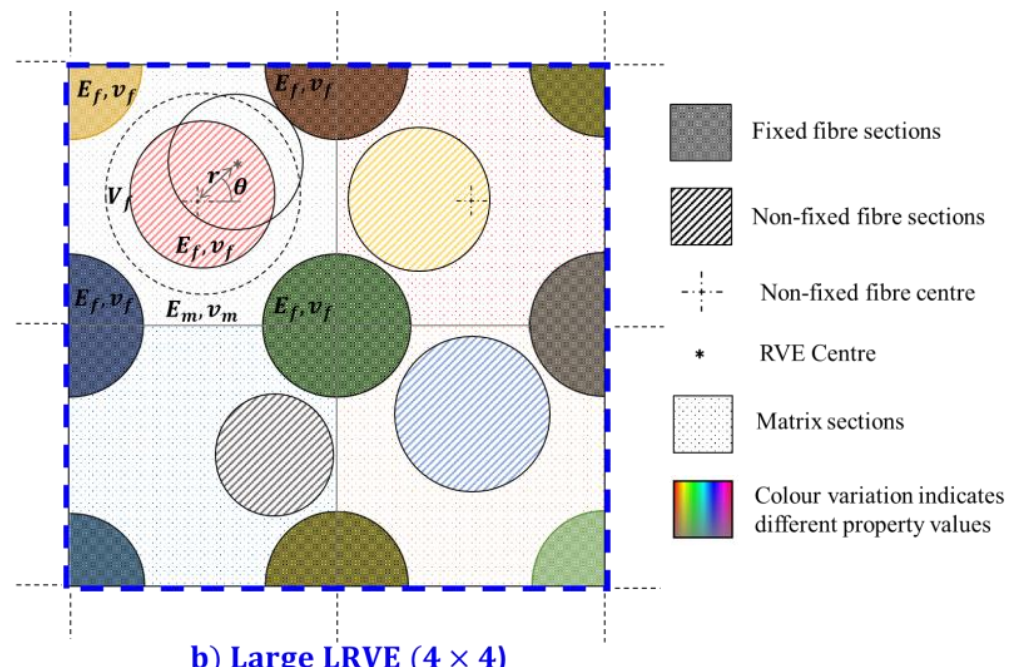

b) Large LRVE $(4 \times 4)$

Figure 6. Illustration of: a) Single RVE uncertainties. b) LRVE uncertainties and fibre properties correlation.

Table 1. Material properties and uncertainties.

\begin{tabular}{|c|c|c|c|c|c|}
\hline \multicolumn{2}{|c|}{ Property/ uncertainty } & Mean/ lower limit & Distribution & CoV/ limits & Categories \\
\hline \multirow{4}{*}{ Fibre (E-glass) } & $E_{f}(\mathrm{GPa})$ & 72.45 & Normal & $5 \%$ & \multirow{6}{*}{ 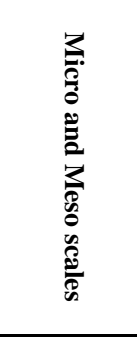 } \\
\hline & $v_{f}$ (ratio) & 0.25 & Normal & $5 \%$ & \\
\hline & Fibre-volume ratio $V_{f}$ & 0.52 & Normal & $5 \%$ & \\
\hline & $\begin{array}{l}\text { Fibre stacking } \\
\quad(r \text { and } \theta)\end{array}$ & RVE centre, $0^{\circ}$ & Uniform & $\begin{array}{l}r: 0-0.08^{*} \\
\theta: 0^{\circ}-360^{\circ}\end{array}$ & \\
\hline \multirow{2}{*}{ Matrix (Epoxy) } & $E_{m}(\mathrm{GPa})$ & 4.0 & Normal & $5 \%$ & \\
\hline & $v_{m}$ (ratio) & 0.3 & Normal & $5 \%$ & \\
\hline \multirow{2}{*}{ Lamina } & $\begin{array}{c}\text { Ply thickness } \\
t_{p l y_{n}}\end{array}$ & $\begin{array}{c}\text { Closed range design } \\
\text { variable } \\
{[0.3 \mathrm{~mm}-0.5 \mathrm{~mm}]} \\
\end{array}$ & Normal & $2.5 \%$ & \multirow{3}{*}{ 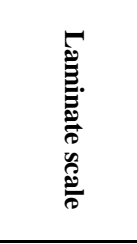 } \\
\hline & $\begin{array}{l}\text { Ply orientation } \\
\theta_{p l y_{n}}\end{array}$ & $\begin{array}{c}\text { Closed range design } \\
\text { variable } \\
{\left[0^{\circ}-90^{\circ}\right]} \\
\end{array}$ & Normal & $2.5^{\circ}$ & \\
\hline \multicolumn{2}{|c|}{ Pressure load (GPa) } & 0.05 & Normal & $5 \%$ & \\
\hline
\end{tabular}

*Fraction of the RVE edge length. 


\subsection{Failure criterion}

As this study focuses on the stiffness response of a composite component, the SLSF is defined by the maximum allowable deformation in the Z-direction $\left(U_{L S}\right)$ that the blades' trailing edge will experience by the pressure load. Hence, the propeller is considered to fail if the deformation exceeds the maximum allowable value. This can be expressed as: $P_{f}=P\left[\left(U_{\text {edge }}-U_{L S}\right) \leq 0\right]$. The position of the selected trailing edge node $U_{\text {edge }}$ is shown in Figure 7.

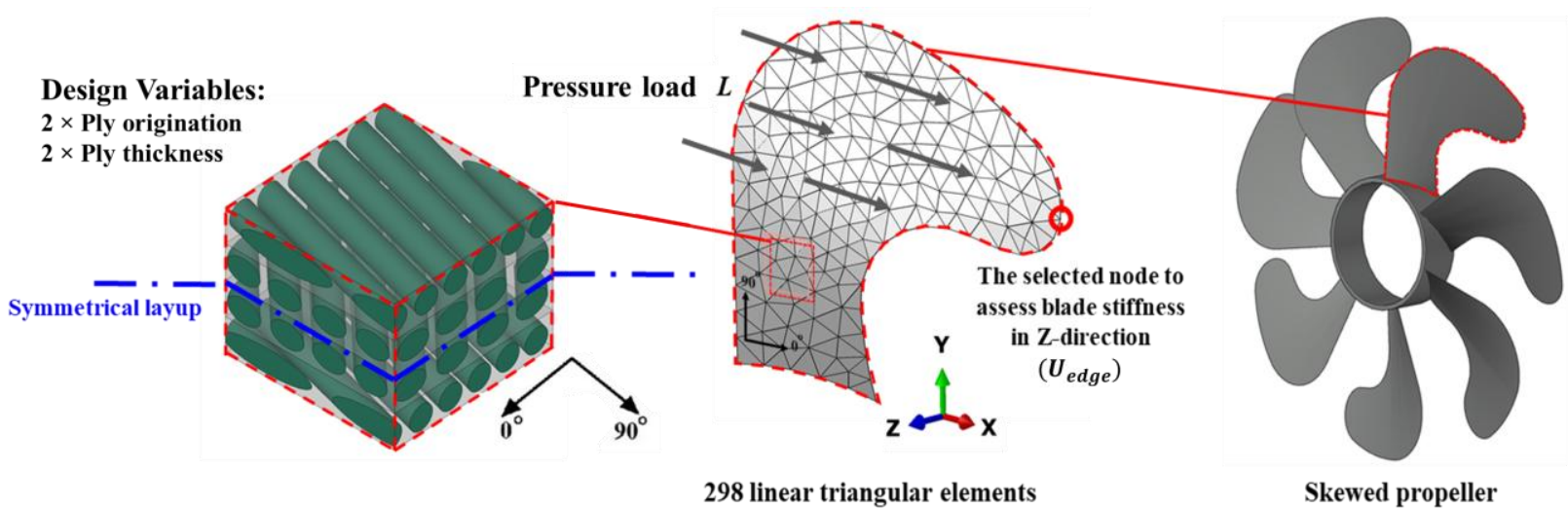

Figure 7. Blade formed from symmetric laminate selected as the RBDO application example.

\subsection{Reliability-based design optimisation process}

The objective of the RBDO problem is to reduce the mass of the propeller blade whilst ensuring that the probability of failure $(\mathrm{PoF})$ remains below 0.05 . Several optimisers could be used to solve the deterministic and probabilistic optimisation problems and the proposed RBDO framework is not limited to a specific optimiser. For this example, the Python Particle Swarm Optimisation pyswarm (PSO) toolbox [38] is integrated within the proposed multi-scale RBDO approach for its robustness and simplicity $[29,39,40]$. The steps required to solve this RBDO problem using the proposed sequential approach, along with the effect of using different LRVE sizes, is detailed below.

\section{5. $1^{\text {st }}$ Deterministic optimisation stage}

The $1^{\text {st }}$ deterministic optimisation is conducted without considering any uncertainties, as shown in Eq. (5):

$$
\begin{aligned}
& \min : \quad M=2 \sum_{n=1}^{2} \mu_{t_{p l y_{n}}} \\
& \text { find: } \quad d_{i}=\left\{\mu_{t_{p l y_{1}}}, \mu_{t_{p l y_{2}}}, \mu_{\theta_{p l y_{1}}}, \mu_{\theta_{p l y_{2}}}\right\} \\
& \text { subject to: } \quad g\left(d_{i}, x_{j}\right)=U_{\text {edge }} \leq 4 \mathrm{~mm} \\
& \left\{d_{1,2} \mid 0.3 \mathrm{~mm} \leq d_{1,2} \leq 0.7 \mathrm{~mm}\right\} \\
& \left\{d_{3,4} \mid 0^{\circ} \leq d_{3,4} \leq 90^{\circ}\right\} \\
& x_{j}=\mu_{x_{j}}
\end{aligned}
$$

Where $M$ is an indicative mass index calculated by summing lamina's thickness values; $\mu_{t_{p l y_{n}}}$ and $\mu_{\theta_{p l y_{n}}}$ are the mean values of the lamina thickness and orientation design variables, respectively. Other inputs, such as loads and material properties, are assumed to be at their mean values.

In order to evaluate the deformation, $U_{\text {edge }}$ and compare it with optimisation constraint in each solution, the optimiser is coupled with the FEA software Abaqus [41], with the settings presented in Table 2. As a result, the optimum design of the $1^{\text {st }}$ deterministic point converged in the $6^{\text {th }}$ optimisation iteration as shown in Figure 8. 
Table 2. PSO settings used in this study.

\begin{tabular}{lcc} 
& \multicolumn{2}{c}{ Values } \\
\cline { 2 - 3 } Settings & $\begin{array}{c}\text { Deterministic } \\
\text { optimisations }\end{array}$ & $\begin{array}{c}\text { Probabilistic } \\
\text { optimisation }\end{array}$ \\
\hline Number of swarm particles & 200 & 50 \\
Max. number of iterations & 10 & 10 \\
Scaling factor to search away from the particle's best-known position & 0.5 & 0.5 \\
Scaling factor to search away from the swarm's best-known position & 0.5 & 0.5 \\
Min. step size of swarm's best position before the search terminates & $10^{-8}$ & $10^{-8}$ \\
Min. change of swarm's best objective value before the search & $10^{-8}$ & $10^{-8}$
\end{tabular}

To assess the stiffness reliability of this design, a statistical distribution is assigned around the design variables, also replacing the deterministic values of all other inputs, namely the homogenised stiffness properties and loading, using the probabilistic data from Table 1 . The homogenised stiffness properties are estimated using the framework presented in Section 2.2, initially using a single, $1 \times 1$ RVE.

The SLSF response is solved numerically, so it is not feasible to assess the PoF without extensive computational power and time. Thus, the PoF is assessed by creating a surrogate model fitted using FEA data points for models created with randomly generated probabilistic inputs around the current optimum design point. In this example, a multivariate nearest-neighbour Python interpolation module scipy.interpolate [42] is used with 1,000 FEA data points to create the surrogate model that estimates the probabilistic stiffness response of the blade, by calculating $U_{\text {edge }}$ values for each sample. The mean error associated with the use of this surrogate model in estimating $U_{\text {edge }}$ is approximately $5.4 \%$. This value is estimated by generating an additional 500 experimental FEA data points and comparing their numerical $U_{\text {edge }}$ values with the values estimated using the surrogate model.

The PoF estimated with the aid of the surrogate model for the $1^{\text {st }}$ design is approximately 0.65 using 40,000 MCS samples. This high PoF value is caused by the fact that the optimisation assumed deterministic inputs at their mean values. In order to shift the design to a safer zone, a second deterministic optimisation is conducted, as discussed in section 3.6, with a reduced allowable design deformation constraint $(3.5 \mathrm{~mm}, 75 \%$ of the global limit state value), as can be seen Figure 8. This value is estimated using Eq. (3) to reach a theoretical PoF of approximately 0.05 in the $2^{\text {nd }}$ deterministic optimum point.

\section{6. $2^{\text {nd }}$ Deterministic optimisation stage}

Based on the assessed reliability, the design is shifted to a more conservative region by reducing the allowable deformation from $4 \mathrm{~mm}$ to $3.5 \mathrm{~mm}$. Although a design point can be selected directly from the previous deterministic optimisation population (data points in red shown in Figure 8), in this instance, a $2^{\text {nd }}$ deterministic optimisation is conducted. However, the design variable ranges are reduced to focus around the region of the updated deflection constraint, as shown by the blue optimisation population in Figure 8, and Eq. (6) where ' indicates the second set of design variables. As a result of using the focused range, the optimiser solution point converged at the $2^{\text {nd }}$ iteration compared with the $6^{\text {th }}$ in the $1^{\text {st }}$ deterministic optimisation.

$$
\begin{array}{ll}
\min : \quad M=2 \sum_{n=1}^{2} \mu_{t_{p l y_{n}}}^{\prime} \\
\text { find: } \quad d^{\prime}{ }_{i}=\left\{\mu_{t_{p l y_{1}}}^{\prime}, \mu_{t_{p l y_{2}}}^{\prime} \mu_{\theta_{p l y_{1}}}^{\prime}, \mu_{\theta_{p l y_{2}}}^{\prime}\right\} \\
\text { subject to: } \quad\left(g\left(d^{\prime}{ }_{i}, x_{j}\right)=U_{\text {edge }}\right) \leq 3.5 \mathrm{~mm} \\
& \left\{d^{\prime}{ }_{1} \mid 0.5 \mathrm{~mm} \leq d^{\prime}{ }_{1} \leq 0.6 \mathrm{~mm}\right\} \\
& \left\{d^{\prime}{ }_{2} \mid 0.4 m m \leq d^{\prime}{ }_{2} \leq 0.5 \mathrm{~mm}\right\} \\
& \left\{d^{\prime}{ }_{3} \mid 60^{\circ} \leq d^{\prime}{ }_{4} \leq 80^{\circ}\right\} \\
& \left\{d^{\prime}{ }_{4} \mid 40^{\circ} \leq d^{\prime}{ }_{4} \leq 60^{\circ}\right\} \\
& x_{j}=\mu_{x_{j}}
\end{array}
$$




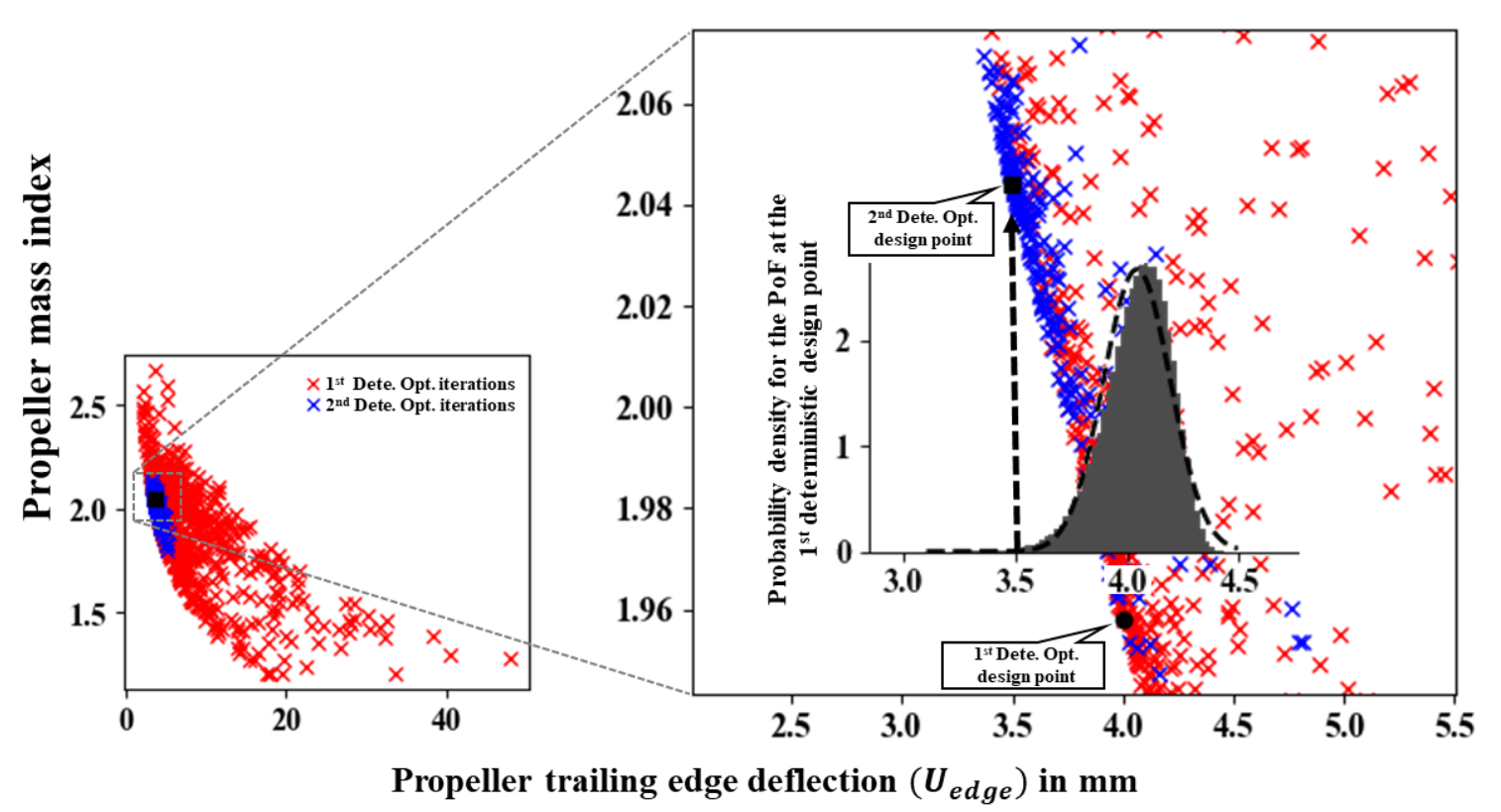

Figure 8. Propeller's $1^{\text {st }}$ and $2^{\text {nd }}$ deterministic optimisation data, showing the design points and the reliability assessment of the $1^{\text {st }}$ design.

\subsection{Probabilistic optimisation stage}

Although the $2^{\text {nd }}$ deterministic optimised solution meets the desired reliability level with a 2.043 mass index, this design can be improved by conducting a probabilistic optimisation that replaces deterministic deformation constraints with a reliability performance level, as presented in Eq. (7):

$$
\begin{aligned}
& \min : \quad M=2 \sum_{n=1}^{2} \mu_{t}^{\prime \prime} p l y_{n} \\
& \text { find: } \quad D_{i}=\left\{\mu_{t}^{\prime \prime}{ }_{p l y_{1}}, \mu_{t}^{\prime \prime}{ }_{p l y_{2}}, \mu_{\theta}^{\prime \prime}{ }_{p l y_{1}}, \mu_{\theta}^{\prime \prime}{ }_{p l y_{2}}\right\} \\
& \text { subject to: } \quad\left(P_{f}=P\left[g\left(D_{i}, x_{j}\right) \leq 4 m m\right]\right) \leq\left(P_{\text {limit }}=0.05\right) \\
& \left\{D_{1,2} \mid d_{1,2}^{\prime}-2 \sigma_{D_{1,2}} \leq D_{1,2} \leq d_{1,2}^{\prime}+2 \sigma_{D_{1,2}}\right\} \\
& \left\{D_{3,4} \mid d_{3,4}^{\prime}-2 \sigma_{D_{3,4}} \leq D_{3,4} \leq d_{3,4}^{\prime}+2 \sigma_{D_{3,4}}\right\} \\
& x_{i}=f\left(x_{i} \mid \mu_{x_{i}}, \sigma_{x_{i}}^{2}\right)
\end{aligned}
$$

Where " indicates the final mean value of each design variable, and the design variables $D_{i}$ feed probabilistically into each reliability MCS iteration, based on their statistical distribution, as shown in Table 1 . On the other hand, it is assumed that the design space is within the boundaries of two standard deviations of the current design $\left(d_{i} \pm 2 \sigma\right)$, as explained in Section 2.5. This range is also used to create an additional 1,000 FEA data points to create the multivariate nearest-neighbour surrogate model, and its accuracy is verified against a set of 500 FEA experimental data points, as explained earlier in Section 2.4. The mean error associated with the surrogate model is $4.8 \%$, with the optimisation settings shown in Table 2 . The final design achieved in this stage converged at the $7^{\text {th }}$ iteration, and showed further mass reduction compared with the $2^{\text {nd }}$ deterministic optimisation with 2.024 mass index, as be seen in Figure 11.

\subsection{Impact of using LRVEs}

The above results are achieved using a single RVE when assessing the stiffness reliability of the component. The single RVE represents various material and geometrical uncertainties, including $V_{f}$ ratio, as shown in Table 1. However, it cannot capture spatial variability in material properties and $V_{f}$ ratio, which can be modelled using LRVEs. The effect of the LRVE size on the reliability assessment of the optimal design from Section 3.7 is now investigated using three LRVE sizes $(2 \times 2,4 \times 4$ and $8 \times 8)$, where a surrogate model for each LRVE size is created to link probabilistic inputs $\left(D_{i}, x_{j}\right)$ with the stiffness response around the optimum design of the single RVE. The use of the LRVEs instead of a single RVE demonstrates a reduced PoF by up to $36 \%$ 
when using an $8 \times 8$ RVE, as can be seen in Figure 9. It indicates that larger RVEs result in a less conservative PoF, due to their proportionally smaller standard deviation, as demonstrated in our previous work [17].

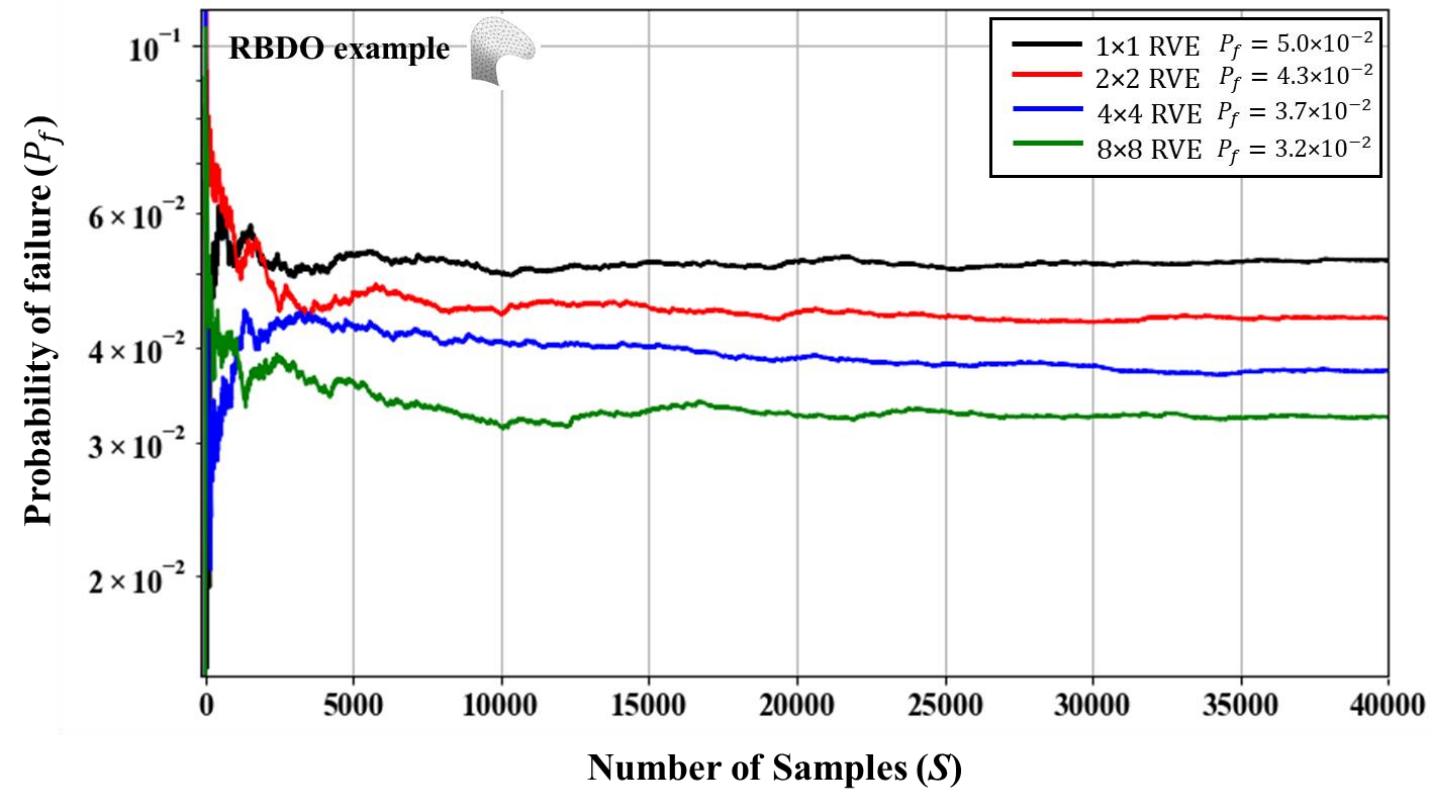

Figure 9. Probability of failure assessment for the final propeller design when using various sizes of LRVEs.

Selecting the suitable RVE size and the boundary conditions depend on many factors such as the fibre size, volume fraction ratio, the thickness of the laminate, loading condition, etc. In this study, it is assumed that the $8 \times 8$ LRVE size is still small enough that periodic boundary conditions can be used. Also, Figure 10 shows that the probability of failure converges as the LRVE size grows.

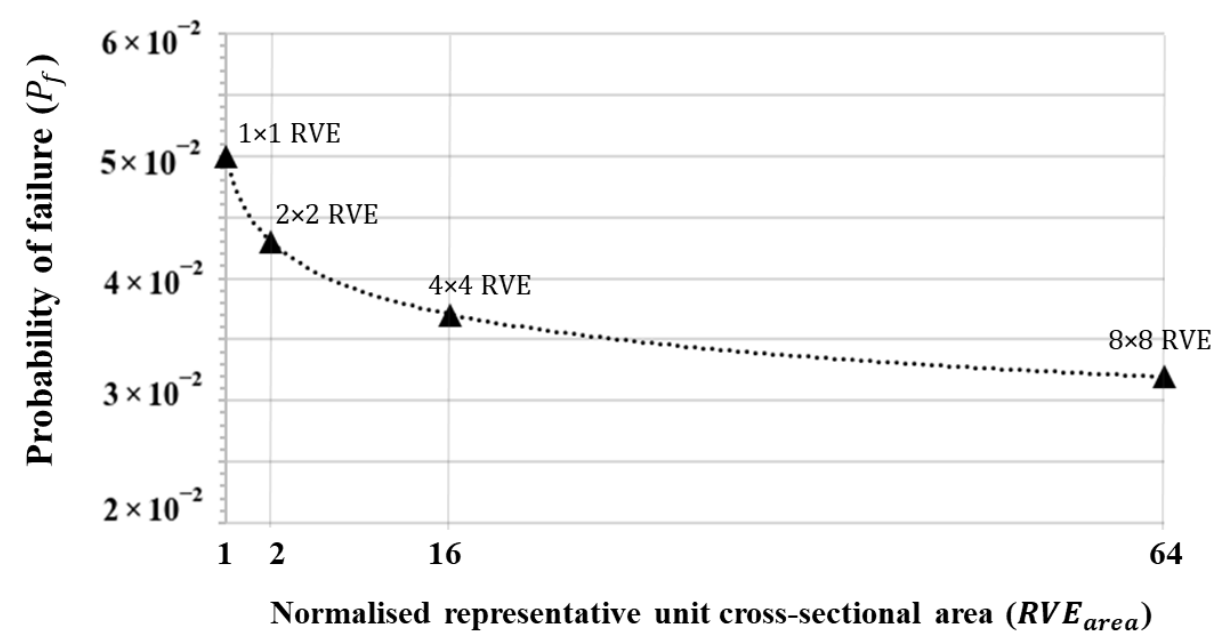

Figure 10. Convergence curve of the probability of failure against the representative unit size.

Improvement in the PoF indicates that designing composites using larger and more realistic representative elements can lead to less conservative designs. Therefore, in addition to assessing the effect of LRVEs on the optimum design obtained with a single RVE, the effect of using LRVEs instead of a single RVE in the probabilistic optimisation stage is investigated. This is achieved by re-running just the probabilistic optimisation stage for each LRVE size individually. The results show that mass reductions of $1.3 \%, 3.0 \%$ and $3.5 \%$ compared with the single RVE are observed using $2 \times 2,4 \times 4$ and $8 \times 8$ LRVEs, respectively, as shown in Figure 
11. This is achieved by a small reduction in the inner ply thickness, along with a reduction in the outer ply angle.

RBDO steps for the composite propellers example:

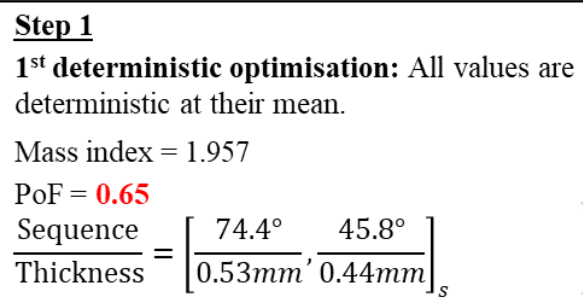

\section{$\underline{\text { Step } 3}$}

Probabilistic optimisation: All uncertainties are considered probabilistically.

\section{Using RVE $1 \times 1$}

Mass index $=2.024$

$\mathrm{PoF}=\mathbf{0 . 0 5 0}$

$\frac{\text { Sequence }}{\text { Thickness }}=\left[\frac{76.8^{\circ}}{0.55 \mathrm{~mm}}, \frac{57.8^{\circ}}{0.46 \mathrm{~mm}}\right]_{S}$

Using RVE $2 \times 2$

Mass index $=1.996$

$\mathrm{PoF}=\mathbf{0 . 0 4 9}$

$\frac{\text { Sequence }}{\text { Thickness }}=\left[\frac{75.2^{\circ}}{0.55 \mathrm{~mm}}, \frac{55.4^{\circ}}{0.44 \mathrm{~mm}}\right]_{S}$ Using RVE 4×4

Mass index $=1.968$

$\mathrm{PoF}=0.049$

$\frac{\text { Sequence }}{\text { Thickness }}=\left[\frac{71.2^{\circ}}{0.55 \mathrm{~mm}}, \frac{57.8^{\circ}}{0.43 \mathrm{~mm}}\right]_{s}$

Using RVE $8 \times 8$

Mass index $=1.953$

$\mathrm{PoF}=0.049$

$\frac{\text { Sequence }}{\text { Thickness }}=\left[\frac{68.7^{\circ}}{0.55 \mathrm{~mm}}, \frac{57.8^{\circ}}{0.42 \mathrm{~mm}}\right]_{S}$
Step 2

$2^{\text {nd }}$ deterministic optimisation: All values are deterministic at their mean.

Mass index $=2.043$

$\mathrm{PoF}=\mathbf{0 . 0 5 0}$

$\frac{\text { Sequence }}{\text { Thickness }}=\left[\frac{73.6^{\circ}}{0.57 \mathrm{~mm}}, \frac{52.8^{\circ}}{0.44 \mathrm{~mm}}\right]_{s}$

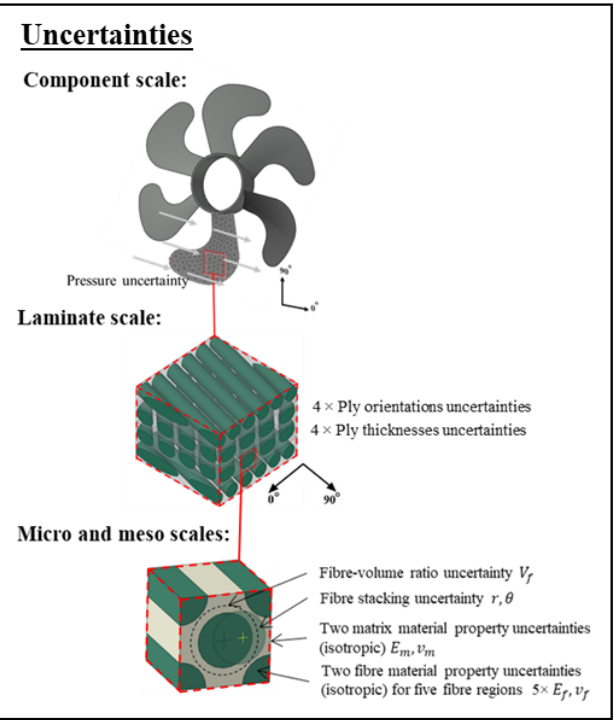

Figure 11. An overview of the propeller example RBDO steps, scope of uncertainties, and the effect of considering different sized LRVEs.

\subsection{Computational efficiency}

The computational efficiency of the proposed multi-scale RBDO framework can be divided into two categories: on-line (or problem-dependent) and off-line (or material dependent). The first category is dependent on the application example and the optimiser used; hence, it is an on-line process, see Figure 4. For this numerical example, a total of 2000 FEA data points were used to create the on-line surrogate models, which took approximately 6 hours' on a standard computer without parallelization. However, the process of generating data points is easily executed in parallel or by analysing more than one blade in the same job and should have near-perfect scaling. 
As for the second category, 40,000 uncertain single RVE and LRVEs are generated to create an off-line library of probabilistic homogenised properties, taking approximately 5 minutes (for each LRVE size), while the generation of the FEA homogenisation data points required to create the surrogate models took approximately 20 hours (using a standard computer without parallelization). It is important to emphasise that the generation of the off-line FEA data points is only needed once (for a certain composite material, i.e. the $E$-glass fibre-epoxy composite in this case), and the resulting surrogate model can be used for various applications and reliability analyses.

The combination of surrogate models reduces the crude MCS reliability analysis time to less than 2 minutes (for 40,000 samples, without parallelisation), which enables the implementation of the RBDO. In contrast, the time required to analyse a single deterministic $8 \times 8$ high fidelity FEA LRVE using the same machine took approximately 26 hours (at least 40,000 of which are needed). Thus, using high-fidelity FEA is not feasible for probabilistic analysis.

Moreover, the sequential approach reduces the number of reliability analyses required to reach the optimum. In total, 357 MCS reliability analyses were required to obtain the final optimum design taking less than 30 mins. The total time for the whole on-line optimisation process for this example which includes the $1^{\text {st }}$ and $2^{\text {nd }}$ deterministic optimisations, corresponding FEA data points, and the probabilistic optimisation is approximately 9 hours' on a standard computer, without parallization. If the same problem was solved using a double-loop approach then a separate reliability analysis may be required for each particle, each iteration. Thus, significantly more MCS analyses would be required, compared with the sequential approach.

\section{Conclusions}

An efficient FEA-based multi-scale reliability framework, previously developed by the authors, is extended and combined with a proposed sequential optimisation strategy to produce an efficient, flexible and accurate RBDO framework for fibre-reinforced composite laminate components. The proposed RBDO strategy is demonstrated by finding the optimum design solution for a composite component under the effect of multi-scale uncertainties, whilst meeting a specific stiffness reliability requirement. Performing this using the double-loop approach is computationally expensive due to the number of uncertainties and function evaluations required to assess the reliability. Thus, a sequential optimisation concept is proposed, which starts by finding a deterministic optimum solution, then assesses the reliability and shifts the constraint limit to a safer region. This is repeated until the desired level of reliability is reached. This is followed by a final probabilistic optimisation to further reduce the mass and meet the desired level of stiffness reliability. In addition, the proposed framework uses several surrogate models to replace expensive FE function evaluations during optimisation and reliability analysis. The numerical example is also used to investigate the effect of using different sizes of LRVEs, compared with a single RVE. In future work, other problem-dependent surrogates such as Kriging will be used to allow predicting lower PoFs with high accuracy.

The main findings are that the integration of the developed multi-scale reliability framework with the sequential RBDO optimisation strategy is proven computationally feasible and it is shown that the use of LRVEs leads to less conservative designs compared with the use of single RVE. This is because the LRVE provides a better representation of the spatial variability of uncertainties in a composite material.

\section{Acknowledgements}

This work was supported by the University of Aberdeen Elphinstone scholarship scheme.

\section{References}

[1] McIlhagger, A., Archer, E. and McIlhagger, R., (2020). 3 - manufacturing processes for composite materials and components for aerospace applications, pp.59-81.

[2] Soutis, C., (2020). 1 - aerospace engineering requirements in building with composites, pp.3-22. 
[3] Yancey, R.N., (2016). Challenges, opportunities, and perspectives on lightweight composite structures: Aerospace versus automotive, In Njuguna, J., (Ed.) Lightweight Composite Structures in TransportWoodhead Publishing, pp. 35-52.

[4] Bloomfield, M.W., Herencia, J.E. and Weaver, P.M., (2009). Enhanced two-level optimization of anisotropic laminated composite plates with strength and buckling constraints, Thin-Walled Structures, 47(11), pp.1161-1167.

[5] Tomar, S.S., Zafar, S., Talha, M., Gao, W. and Hui, D., (2018). State of the art of composite structures in non-deterministic framework: A review, Thin-Walled Structures, 132pp.700-716.

[6] Agarwal, H., Renaud, J.E., Preston, E.L. and Padmanabhan, D., (2004). Uncertainty quantification using evidence theory in multidisciplinary design optimization, Reliability Engineering \& System Safety, 85(1), pp.281-294.

[7] Sriramula, S. and Chryssanthopoulos, M.K., (2009). Quantification of uncertainty modelling in stochastic analysis of FRP composites, Composites Part A: Applied Science and Manufacturing, 40(11), pp.16731684 .

[8] Antonio, C., Soeiro, A.,V. and Marques, A.,T., (1993). Optimization in reliability based design of laminated composite structures, WIT Transactions on the Built Environment, 2

[9] Yang, L. and Ma, Z.K., (1990). Optimum design based on reliability for a composite structural system, Computers \& Structures, 36(5), pp.785-790.

[10] Deo, S. and Rais-Rohani, M., (1999). Reliability-Based Design of Composite Sandwich Plates with NonUniform Boundary Conditions.

[11] Rais-Rohani, M. and Singh, M.N., (2004). Comparison of global and local response surface techniques in reliability-based optimization of composite structures, Structural and Multidisciplinary Optimization, 26 (5), pp.333-345.

[12] Thompson, M., Eamon, C. and Rais-Rohani, M., (2006). Reliability-Based Optimization of FiberReinforced Polymer Composite Bridge Deck Panels, Journal of Structural Engineering-Asce - J STRUCT ENG-ASCE, 132

[13] Dey, S., Mukhopadhyay, T., Khodaparast, H. and Adhikari, S., (2015). A Response Surface Modelling Approach for Resonance Driven Reliability Based Optimization of Composite Shells, Periodica Polytechnica Civil Engineering, 60

[14] Rouhi, M. and Rais-Rohani, M., (2013). Modeling and probabilistic design optimization of a nanofiberenhanced composite cylinder for buckling, Composite Structures, 95pp.346-353.

[15] L. Wang, Q. Ren, Y. Ma and D. Wu, (2019). Optimal maintenance design-oriented nonprobabilistic reliability methodology for existing structures under static and dynamic mixed uncertainties, $\mathbf{6 8}(2)$, pp.496-513.

[16] Shi, B., Den, Z., Tan, L., Zhao, Y. and Zhang, X., (2020). Micromechanics-based reliability analysis method for laminated composite structures, Mechanics of Advanced Materials and Structures, pp.1-18.

[17] Omairey, S.L., Dunning, P.D. and Sriramula, S., (2019). Multiscale surrogate-based framework for reliability analysis of unidirectional FRP composites, Composites Part B: Engineering, 173pp.106925.

[18] Omairey, S.L., Dunning, P.D. and Sriramula, S., (2018). Influence of micro-scale uncertainties on the reliability of fibre-matrix composites, Composite Structures, 203pp.204-216. 
[19] Zhou, X., Gosling, P.D., Ullah, Z., Kaczmarczyk, Ł and Pearce, C.J., (2016). Exploiting the benefits of multi-scale analysis in reliability analysis for composite structures, Composite Structures, 155 pp.197-212.

[20] Shaw, A., Sriramula, S., Gosling, P.D. and Chryssanthopoulos, M.K., (2010). A critical reliability evaluation of fibre reinforced composite materials based on probabilistic micro and macro-mechanical analysis, Composites Part B: Engineering, 41 (6), pp.446-453.

[21] Ghasemi, H., Rafiee, R., Zhuang, X., Muthu, J. and Rabczuk, T., (2014). Uncertainties propagation in metamodel-based probabilistic optimization of CNT/polymer composite structure using stochastic multiscale modeling, Computational Materials Science, 85pp.295-305.

[22] Ma, X., Tian, K., Li, H., Zhou, Y., Hao, P. and Wang, B., (2020). Concurrent multi-scale optimization of hybrid composite plates and shells for vibration, Composite Structures, 233pp.111635.

[23] Wang, L., Cai, Y. and Liu, D., (2018). Multiscale reliability-based topology optimization methodology for truss-like microstructures with unknown-but-bounded uncertainties, Computer Methods in Applied Mechanics and Engineering, 339pp.358-388.

[24] Díaz, J., Cid Montoya, M. and Hernández, S., (2016). Efficient methodologies for reliability-based design optimization of composite panels, Advances in Engineering Software, 93pp.9-21.

[25] Moustapha, M. and Sudret, B., (2019). Surrogate-assisted reliability-based design optimization: a survey and a unified modular framework, Structural and Multidisciplinary Optimization, 60 (5), pp.2157-2176.

[26] Peng, X., Guo, Y., Qiu, C., Wu, H., Li, J., Chen, G., Jiang, S. and Liu, Z., (2020). Reliability optimization design for composite laminated plate considering multiple types of uncertain parameters, Engineering Optimization, pp.1-16.

[27] Li, X., Gong, C., Gu, L., Jing, Z., Fang, H. and Gao, R., (2019). A reliability-based optimization method using sequential surrogate model and Monte Carlo simulation, Structural and Multidisciplinary Optimization, 59 (2), pp.439-460.

[28] Lopez, R.H., Lemosse, D., de Cursi, J.,Eduardo Souza, Rojas, J. and El-Hami, A., (2011). An approach for the reliability based design optimization of laminated composites, Engineering Optimization, 43 (10), pp.1079-1094.

[29] Chen, J., Ge, R. and Wei, J., (2008). Probabilistic optimal design of laminates using improved particle swarm optimization, Engineering Optimization, 40 (8), pp.695-708.

[30] López, C., Bacarreza, O., Baldomir, A., Hernández, S. and H. Ferri Aliabadi, M., (2017). Reliability-based design optimization of composite stiffened panels in post-buckling regime, Structural and Multidisciplinary Optimization, 55(3) pp.1121-1141.

[31] Dejaegher, B. and Heyden, Y.V., (2009). 1.17 - sequential optimization methods, pp.547-575.

[32] Du, X. and Chen, W., (2004). Sequential Optimization and Reliability Assessment Method for Efficient Probabilistic Design, Journal of Mechanical Design, 126 (2), pp.225-233.

[33] Sohouli, A., Yildiz, M. and Suleman, A., (2018). Efficient strategies for reliability-based design optimization of variable stiffness composite structures, Structural and Multidisciplinary Optimization, 57 (2), pp.689-704.

[34] Xia, Z., Zhang, Y. and Ellyin, F., (2003). A unified periodical boundary conditions for representative volume elements of composites and applications, International Journal of Solids and Structures, 40(8), pp.1907-1921. 
[35] Sakata, S., Ashida, F. and Enya, K., (2012). A Microscopic failure probability analysis of a unidirectional fiber reinforced composite material via a multiscale stochastic stress analysis for a microscopic random variation of an elastic property, Computational Materials Science, 62 pp.35-46.

[36] Henrik, S.T., Branner, K., Leon Mishnaevsky, J. and John, D.S., (2013). Uncertainty modelling and code calibration for composite materials, Journal of Composite Materials, 47 (14), pp.1729-1747.

[37] Geers, M.G.D., Kouznetsova, V.G. and Brekelmans, W.A.M., (2010). Multi-scale computational homogenization: Trends and challenges, Journal of Computational and Applied Mathematics, 234 (7), pp.2175-2182.

[38] Lee, A., (2014). pyswarm, Available at: https://pypi.org/project/pyswarm/

[39] Windarto, Eridani and Purwati, U.D., (2019). A comparison of continuous genetic algorithm and particle swarm optimization in parameter estimation of Gompertz growth model, AIP Conference Proceedings, 2084 (1), pp.020017.

[40] D. W. Boeringer and D. H. Werner, (2004). Particle swarm optimization versus genetic algorithms for phased array synthesis, 52(3), pp.771-779.

[41] ABAQUS Inc., (2013). Abaqus CAE, 2017

[42] T. E. Oliphant, (2007). Python for Scientific Computing, Computing in Science \& Engineering, 9 (3), pp.10-20.

\section{Appendixes}

\section{Appendix A}

A code extract showing the process used to generate random fibres staked within each RVE:

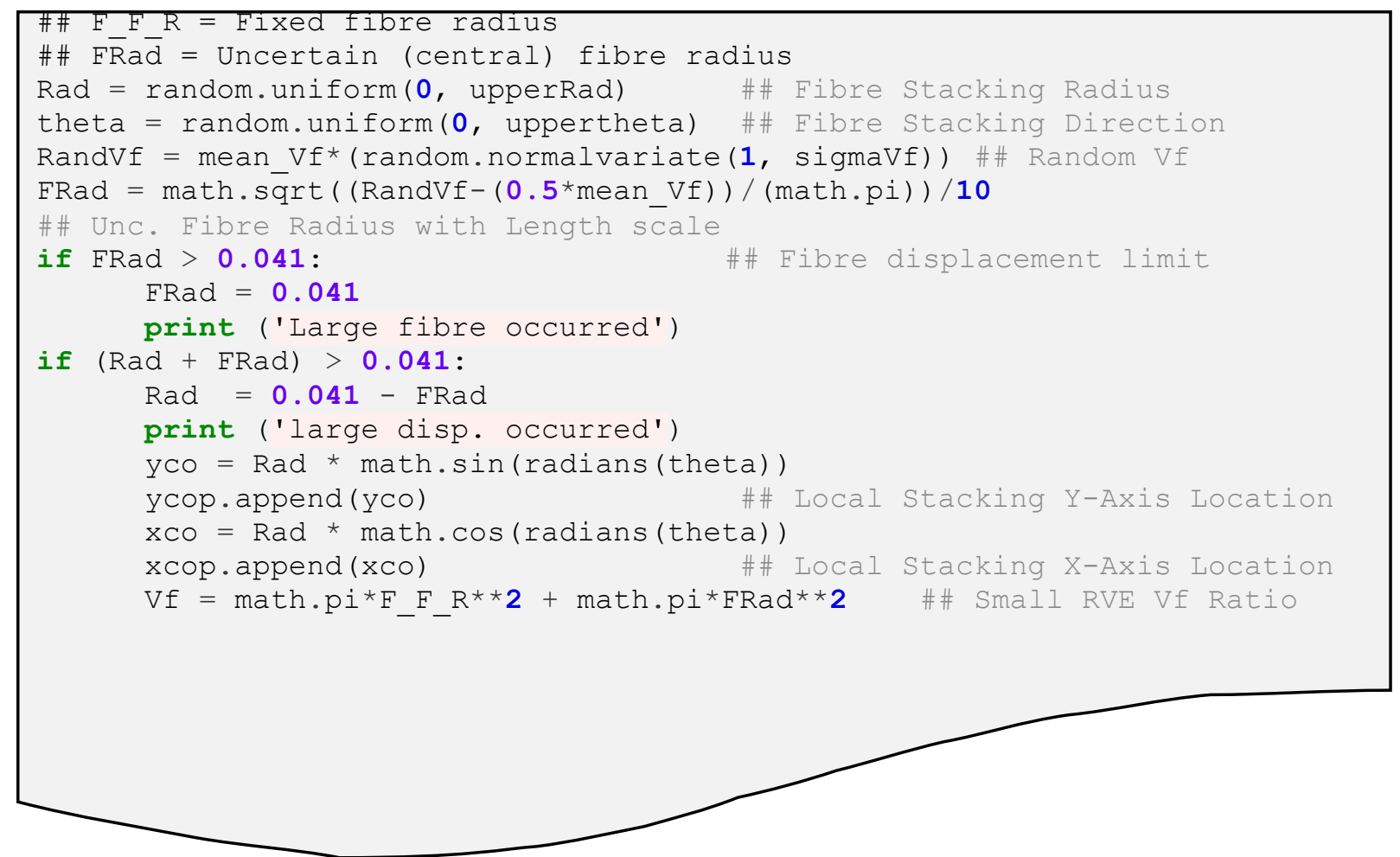




\section{Appendix B}

The code developed for smoothing the random matrix property values using the blur kernel:

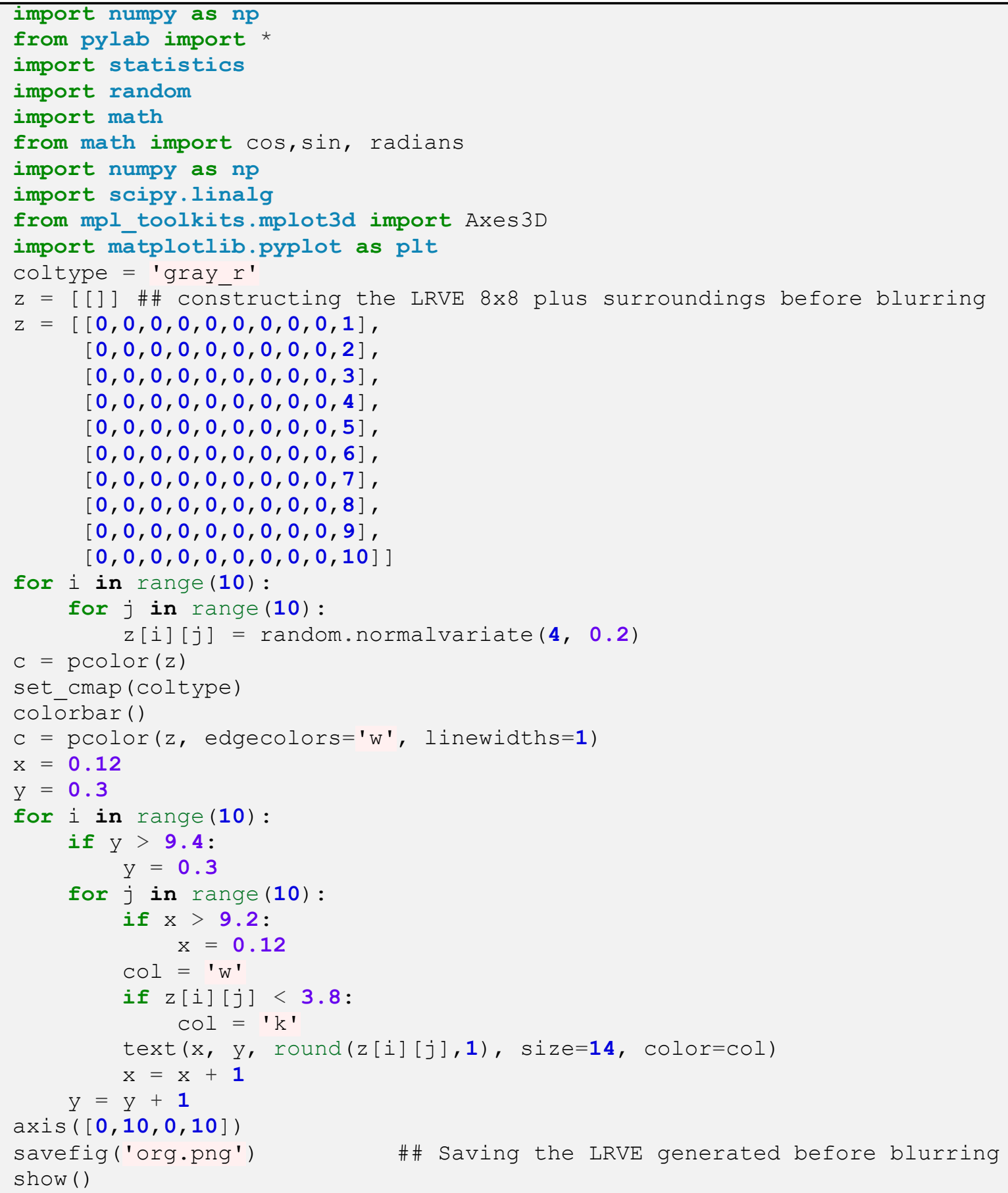




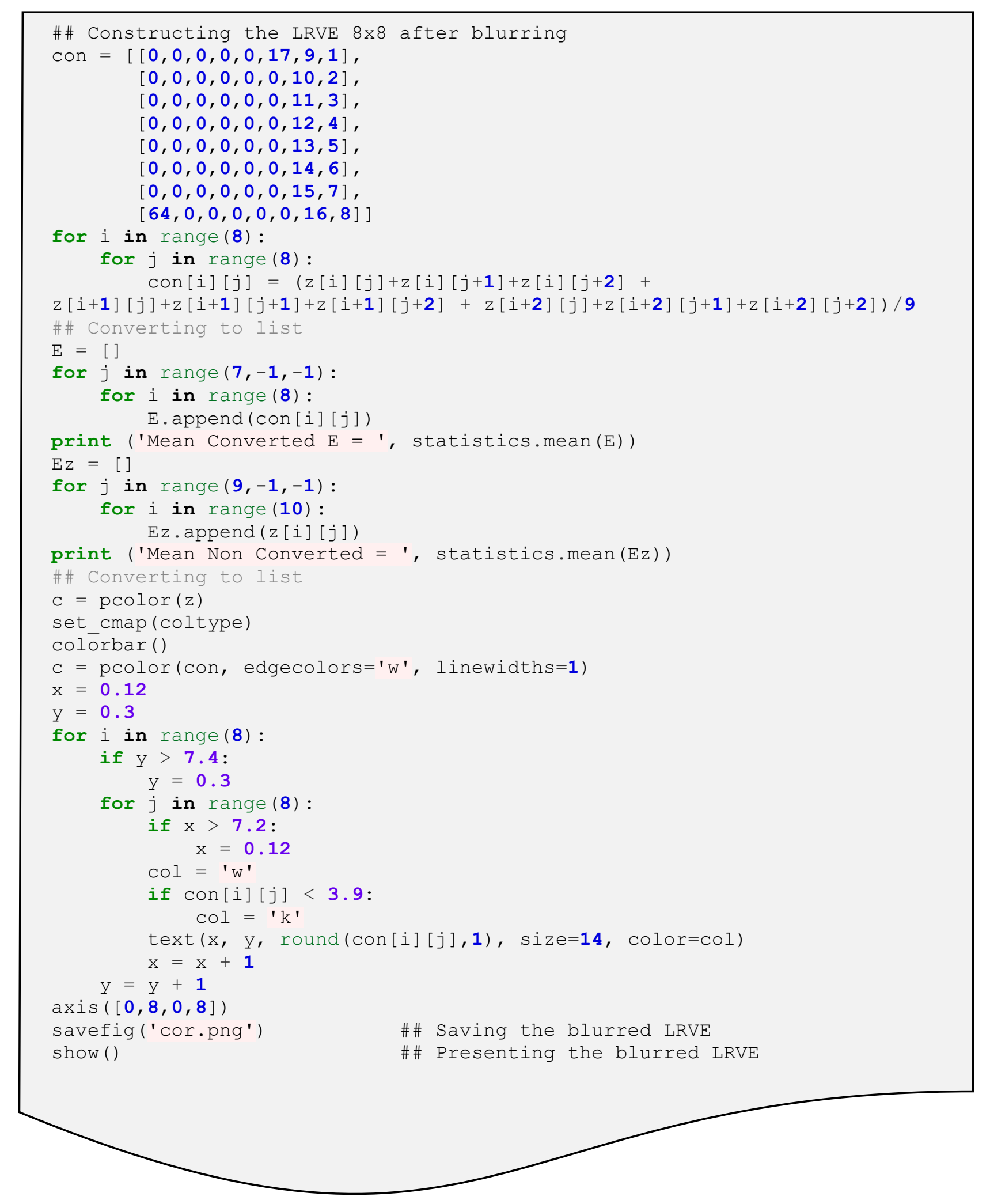

